\title{
Inverse Association of Serum Adipsin with the Remission of Nonalcoholic Fatty-Liver Disease: A 3-Year Community-Based Cohort Study
}

\author{
Yingying Gu ${ }^{a, b}$ Jing Luo ${ }^{a, b, c}$ Qian Chen ${ }^{a, b, d}$ Yun Qiu, a, Yujia Zhou ${ }^{a, b}$

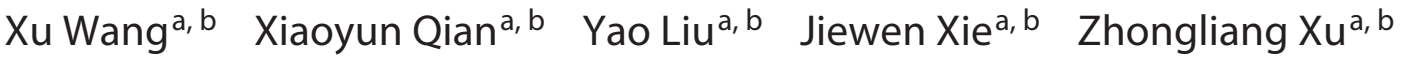 \\ Wenhua Ling ${ }^{a, b}$ Yuming Chen ${ }^{b, e}$ Lili Yang ${ }^{a, b}$ \\ aDepartment of Nutrition, School of Public Health, Sun Yat-Sen University (Northern Campus), Guangzhou, China; \\ ${ }^{b}$ Guangdong Provincial Key Laboratory of Food, Nutrition and Health, Guangzhou, China; 'Huaian Center for \\ Disease Prevention and Control, Huaian, China; ${ }^{\mathrm{d} D e p a r t m e n t}$ of Cardiology, Sun Yat-Sen Memorial Hospital, Sun \\ Yat-Sen University, Guangzhou, China; 'Department of Medical Statistics and Epidemiology, School of Public \\ Health, Sun Yat-Sen University (Northern Campus), Guangzhou, China
}

\section{Keywords}

Prospective cohort study · Metabolic disease · Adipokine · Adipose tissue $\cdot$ Complement factor

\section{Abstract \\ Purposes: Adipokine alterations contribute to the develop- ment and remission of nonalcoholic fatty-liver disease (NAFLD). Adipsin is one of the most abundant adipokines and is almost exclusively produced by adipocytes. However, data on adipsin in human NAFLD are limited and controver- sial. We performed this study to investigate the association between adipsin and the remission of NAFLD in middle- aged and elderly Chinese adults. Methods: Whether adipsin is associated with the remission of NAFLD in a 3-year com- munity-based prospective cohort study was investigated. Baseline levels of adipsin were measured in serum samples collected from 908 NAFLD participants. NAFLD was diag- nosed using abdominal ultrasonography. Logistic regres- sion analysis and a multiple stepwise logistic regression model including different variables were conducted to eval- uate the association between serum adipsin levels and the}

karger@karger.com www.karger.com/anm

Karger $\stackrel{\text { ' }}{5}$
(C) 2021 The Author(s)

Published by S. Karger AG, Basel

This article is licensed under the Creative Commons Attribution 4.0 International License (CC BY) (http://www.karger.com/Services/ OpenAccessLicense). Usage, derivative works and distribution are permitted provided that proper credit is given to the author and the original publisher. remission of NAFLD. Results: During a mean follow-up of $3.14 \pm 0.36$ years, $247(27.20 \%)$ participants with NAFLD at baseline were in remission. At baseline, serum adipsin concentration was positively correlated with body mass index ( $r$ : $0.39, p<0.001)$, insulin $(r: 0.31, p<0.001)$, and homeostasis model assessment of insulin resistance $(r: 0.31, p<0.001)$ and was inversely associated with NAFLD remission with a fully adjusted odds ratio (OR) of $0.28(0.16-0.48)$ ( $p$ trend $<0.001$ ). In a multiple stepwise logistic regression model, circulating adipsin independently predicted NAFLD remission (OR: $0.284,95 \%$ confidence interval [Cl]: $0.172-0.471, p$ for trend $<0.001)$. The area under the receiver operating characteristic curve was 0.751 ( $95 \% \mathrm{Cl}: 0.717-0.785)(p<0.001)$ for the prediction model of NAFLD remission. Conclusions: We provide evidence for an association between serum adipsin levels and the remission of NAFLD in a community-based prospective cohort study. Serum adipsin can be a potential biomarker for predicting NAFLD remission.

(C) 2021 The Author(s)

Published by S. Karger AG, Basel

Yingying Gu and Jing Luo contributed equally to this manuscript.
Correspondence to:

Yuming Chen, chenyum@mail.sysu.edu.cn

Lili Yang, yangll7@ mail.sysu.edu.cn 


\section{Introduction}

Nonalcoholic fatty-liver disease (NAFLD) has been a major cause of chronic liver disease worldwide, with an estimated overall global prevalence of $25.24 \%$ [1]. NAFLD is characterized by the presence of hepatic steatosis (the presence of $\geq 5 \%$ hepatic steatosis) on liver histology in individuals with no history of significant alcohol consumption and no other known secondary causes of hepatic fat accumulation [2]. As a spectrum of disease states, NAFLD starts as simple liver steatosis (SS), which may progress to nonalcoholic steatohepatitis (NASH), advanced fibrosis, and even hepatocellular carcinoma [3]. NAFLD is a dynamic condition, and early-stage NAFLD, for example, simple steatosis, can remit to a normal liver, but once it develops to NASH, the risk leading to liver cancer or death caused by NAFLD increases dramatically [4]. Hence, it is important to identify circulating factors associated with NAFLD remission, which can be used as biomarkers for NAFLD prognosis.

Adipose tissue, as an active endocrine organ and the main site for the storage of excess energy in the form of triglycerides (TGs), participates in energy metabolism [5, 6]. Adipose tissue secretes a number of adipokines, many of which are involved in energy homeostasis and inflammation. Dysregulated production of adipokines profoundly affects the function of different organs and contributes to the development of various metabolic diseases, including insulin resistance (IR), dyslipidemia, and NAFLD [7]. Adipsin is one of the most abundant adipokines mainly produced by adipose tissue as the first adipokine described [8]. Although the physiological effect of adipsin remains largely unknown, studies have found that this adipokine can stimulate synthesis of TGs which contributes to development of NAFLD [9-11]. Adipsin is also known as adipocyte complement factor $\mathrm{D}$, which catalyzes the rate-limiting step in the alternative pathway of complement activation [12, 13]. Growing evidence suggests the presence of a connection between the complement system and development of obesity, inflammation, and IR [11, 14, 15]. Rensen et al. [16] reported that widespread activation of the complement system in NAFLD is increased with disease severity [17].

Population-based studies have demonstrated that circulating adipsin levels are significantly elevated in obese individuals and postmenopausal women [18-20]. It was reported that higher adipsin concentrations were independently associated with greater odds of obesity. However, human studies on the association between adipsin levels and NAFLD are limited and have yielded conflict- ing results. Similar adipsin levels were observed between NAFLD participants and controls, and no difference was found between SS and NASH in a pediatric population $[21,22]$. In our previous case-control study including 211 Chinese participants, we observed that circulating adipsin levels were independently positively associated with the occurrence of NAFLD [23]. To further evaluate the association between adipsin levels and NAFLD disease remission, we evaluated serum adipsin levels in 908 NAFLD participants with a 3-year prospective follow-up in middle-aged and elderly Chinese adults.

\section{Participants and Methods}

\section{Participants}

The current study was based on the Guangzhou Nutrition and Health Study (GNHS), a community-based prospective cohort study in China, which aimed to identify determinants of common chronic diseases. Participants were residents in Guangzhou and were recruited from communities in Guangzhou through advertisements, health talks, and referrals. For the cohort, participants with the following conditions were included: 40-80 years of age at baseline, living in Guangzhou for at least 5 years, and Chinese people and participants with any of the following conditions were excluded: had a history of hospital-confirmed failure(s) of heart, liver, or kidney, cancer, CVD events, metabolic bone diseases, glucocorticoid use (over 3 months) or sexual hormone use (over 6 months), and spine or hip fractures; on a special diet due to a disease or weight control; mental and physical disability; likely to move to another city within 5 years; and did not want to attend any one item of the survey or sample collection. The process and timeline applied in this prospective cohort study are shown in Figure 1. From September 2008 to February 2010, a total of 3,169 eligible Chinese adults of 40-75 years of age were recruited. Of these, 2,510 participants were included in the first follow-up survey between April 2011 and March 2013. An additional 879 participants were recruited between March 2013 and November 2013 to account for participant attrition, resulting in 2,945 participants included in the second follow-up survey at a mean of $3.09 \pm 0.41$ years between April 2014 and May 2017. In all the follow-up phases, all participants underwent a comprehensive questionnaire survey and body assessment, including physical examination, routine biochemical blood analyses, and hepatitis virus and human immunodeficiency virus tests. Details of the questionnaire data collection have been described previously [24]. Participants with any of the following conditions were excluded from the analysis: participants without NAFLD, excessive alcohol consumption $(\geq 140 \mathrm{~g} /$ weeks in men or $\geq 70 \mathrm{~g}$ /weeks in women), the presence of secondary causes of hepatic fat accumulation (e.g., viral hepatitis, lipodystrophy, parenteral nutrition, Wilson's disease, inborn errors of metabolism, and long-term use of steatogenic medications) [2], women who were pregnant or nursing, and severe diseases (e.g., cancer, stroke, renal dysfunction, and heart failure). Participants who had missing data on the blood biochemical index and abdominal ultrasonographic results were also excluded from the present study. Ultimately, 908 participants with baseline serum adipsin measurements were in- 


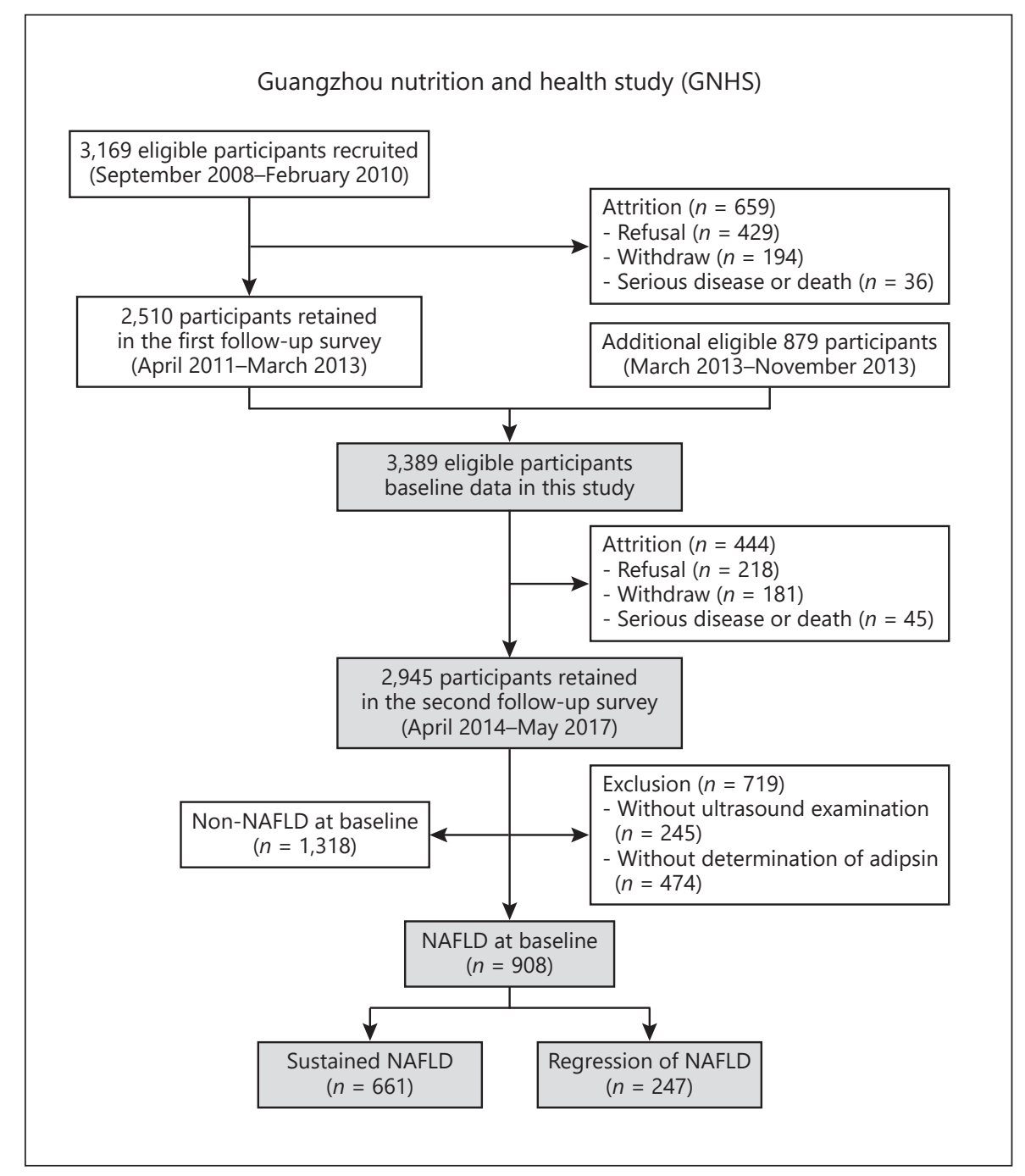

Fig. 1. Procedure of enrollment of patients in the present study. NAFLD, nonalcoholic fatty-liver disease.

cluded in the analysis and divided into 2 groups as persisting NAFLD and NAFLD remission according to NAFLD status after the 3-year follow-up. Since the first follow-up, we began to conduct abdominal ultrasound examinations in this cohort. Thus, in the present study, our "baseline data" were derived from the first follow-up survey, and the "follow-up data" were based on the second follow-up survey.

The GNHS was registered on ClinicalTrials.gov (registration number NCT03179657) and approved by the Ethics Committee of the School of Public Health at Sun Yat-sen University (approval number ZDGWYL2009-3), which meets the guidelines laid down in the Declaration of Helsinki. Written informed consent was obtained from all participants.

\section{Clinical and Laboratory Assessment}

Trained interviewers conducted face-to-face and one-by-one interviews with all participants to complete a detailed survey including questions on demographic characteristics, socioeconomic status, behavior and lifestyle (e.g., alcohol drinking, smoking, and physical activity), histories of chronic diseases, and current medi- cation use through standardized questionnaires. Participants who drank alcohol once a week or smoked at least one cigarette per day for at least 6 months were identified as current drinkers or smokers.

Height, weight, waist circumference, and hip circumference were measured twice with the participants standing and wearing light daily clothing, and the mean values were calculated. Body mass index (BMI) was calculated as weight $(\mathrm{kg})$ divided by the height squared $\left(\mathrm{m}^{2}\right)$, and values greater than $24 \mathrm{~kg} / \mathrm{m}^{2}$ were identified as overweight [25]. The waist-to-hip ratio (WHR) was calculated as the waist circumference divided by the hip circumference. Blood pressure was measured in duplicate on the left arm using an automated sphygmomanometer (HEM 7011; OMRON Corp., Osaka, Japan) and averaged for analyses. The metabolic equivalent intensity was calculated to estimate daily physical activity using a 24-h physical activity questionnaire [26]. Dual-energy X-ray absorptiometry scans (Discovery W; Hologic Inc., Waltham, MA, USA) were used to quantify the fat mass of the trunk region.

Blood samples from all participants were collected via venipuncture after an overnight fast of $8 \mathrm{~h}$ or more, and the serum was 
collected within $2 \mathrm{~h}$ afterward. Serum samples were stored at $-80^{\circ} \mathrm{C}$ until analysis. The baseline biochemical measurements detected enzymatically by a Hitachi 7600-010 automated analyzer (Hitachi, Tokyo, Japan) included TGs, total cholesterol, albumin (Alb), high-density lipoprotein cholesterol (HDL-C), low-density lipoprotein cholesterol (LDL-C), uric acid (UA), aspartate aminotransferase (AST), alanine aminotransferase (ALT), fasting glucose, and insulin. The homeostasis model assessment of IR $($ HOMA-IR) was calculated as fasting glucose $(\mathrm{mmol} / \mathrm{L}) \times$ fasting insulin $(\mu \mathrm{U} / \mathrm{mL}) / 22.5$. Adipsin levels were measured by quantitative sandwich enzyme immunoassay using kits obtained from R\&D Systems (DFD00, Minneapolis, MN, USA) with a Spark 10M Multimode Reader Platform (Tecan Trading AG, Männedorf, Switzerland).

\section{Evaluation of NAFLD}

NAFLD was diagnosed by abdominal ultrasonography following the standard criteria issued by the Fatty Liver Disease Study Group of the Chinese Liver Disease Association [25, 26]. The remission of NAFLD was defined as the presence of NAFLD at baseline and the absence at the follow-up. Ultrasound analyses were performed by 2 experienced technicians who were blinded to the participants' data on a Doppler sonography machine (Sonoscape SSI-5500, Shenzhen, China) with a 3.5-MHz probe. Between-operator reliability evaluation for ultrasonography was assessed in 100 participants, showing very good precision (Spearman's $r=$ 0.911 , kappa $=0.875$, consistency rate $=93 \%, p<0.001)$. We estimated the validity evaluation of NAFLD in 34 participants who further underwent CT scanning, with the radiologists blinded to the ultrasound results. There was good agreement in diagnosing NAFLD between the 2 diagnostic methods (Spearman's $r=0.905$, kappa $=0.691$, consistency rate $=85 \%, p<0.001$ ). The same experienced technician performed ultrasonography at the baseline and follow-up exams. Follow-up evaluations of hepatic ultrasonography were similarly conducted following the standard protocol.

\section{Statistical Analysis}

Continuous variables were reported as the means and standard deviations for normally distributed variables or as medians and interquartile ranges for non-normally distributed variables. Categorical variables were summarized as numbers and percentages. The adipsin level was treated as a categorical variable by quartiles. Baseline demographic and laboratory characteristics of the participants according to the status of NAFLD remission or quartiles of adipsin levels were compared using the independent samples $t$ test, one-way ANOVA test, Kruskal-Wallis, and $\chi^{2}$ test, where appropriate. Tukey post hoc tests were used to follow one-way ANOVA to further define differences. The correlation of serum adipsin levels with other demographic and laboratory characteristics at baseline was evaluated by Spearman's correlation analysis and partial correlation analysis. The multivariable logistic regression models were adjusted for metabolic risk factors. We adjusted for sex and age in model 1. To investigate the independent associations, we further adjusted for BMI, physical activity (metabolic equivalent), current smoking and drinking, hypertension, and diabetes in model 2 plus HOMA-IR, Alb, TGs, LDL-C/HDL-C ratio, $\mathrm{UA}$, and AST/ALT ratio in model 3. All 3 models were applied to the subgroup analysis as described above. Furthermore, multiple stepwise logistic regression analyses were performed to determine the independent predictors and the model for predicting NAFLD remission. We evaluated the prediction accuracy of the model built by multiple stepwise logistic regression analysis with area under the receiver operating characteristic curve (AUROC) with 95\% confidence interval (CI). Sensitivity, specificity, negative likelihood ratio, and positive likelihood ratio of this model were calculated. To test for selection bias, NAFLD participants included in the present study were compared to those who failed to complete the follow-up survey or were lost to the follow-up. All statistical procedures were performed using SPSS Statistics software (version 22.0; SPSS Inc., Chicago, IL, USA). A significant difference was considered when a 2 -tailed $p$ value was $<0.05$.

\section{Results}

\section{Baseline Characteristics of the Participants}

As shown in Figure 1, 908 participants with baseline serum adipsin measurements were ultimately included in the analysis. Except for LDL-C and ALT, no significant differences were observed in major demographic, anthropometric, or laboratory variables when NAFLD participants were included in the present study compared to those who were lost to the follow-up. The mean follow-up time was $3.14 \pm 0.36$ years. The remission rate of NAFLD during this period was $27.20 \%$ (247/908). The baseline characteristics of the NAFLD participants are shown in Table 1. At baseline, participants with subsequently persisting NAFLD had a median age of 59.96 years with $31.6 \%$ men, and those with NAFLD remission had a median age of 59.50 years with $31.2 \%$ men. The baseline levels of BMI, WHR, systolic blood pressure, diastolic blood pressure, fasting glucose, insulin, HOMA-IR, TGs, UA, ALT, and trunk fat percentage were significantly lower, while the HDL-C level was significantly higher in participants with NAFLD remission (all $p<0.05$ ). There was no significant difference between participants with persisting NAFLD and NAFLD remission for the use of antihypertensive agents, hypoglycemic agents, and lipidlowering agents.

\section{Adipsin Levels of NAFLD Participants with or without} NAFLD Remission at the Follow-Up

As shown in Figure 2a, baseline adipsin levels in participants with persisting NAFLD (3,565.52, 3,203.18$4,020.48 \mathrm{ng} / \mathrm{mL})$ were higher than those in remission $(3,251.33,2,956.20-3,628.15 \mathrm{ng} / \mathrm{mL})(p<0.001)$. Meanwhile, the results were consistent in subgroups when stratified by age, sex, BMI, HOMA-IR, TGs, and UA (all $p$ values $<0.01$ ) (Fig. 2).

All participants were divided into 4 groups according to the quartiles of serum adipsin levels. Online supple- 
Table 1. Baseline characteristics by the remission of NAFLD

\begin{tabular}{|c|c|c|c|c|}
\hline Variables & $\begin{array}{l}\text { Total } \\
(n=908)\end{array}$ & $\begin{array}{l}\text { Persisting NAFLD } \\
(n=661)\end{array}$ & $\begin{array}{l}\text { NAFLD remission } \\
(n=247)\end{array}$ & $\begin{array}{l}p \text { value } \\
\text { (persisting vs. } \\
\text { NAFLD remission) }\end{array}$ \\
\hline Sex (males), ${ }^{\dagger} n(\%)$ & $286(31.5)$ & $209(31.6)$ & $77(31.2)$ & 0.898 \\
\hline Adipsin, $\mathrm{ng} / \mathrm{mL}^{\S}$ & $3,469.35(3,111.51-3,920.78)$ & $3,565.52(3,203.18-4,020.48)$ & $3,251.33(2,956.20-3,628.15)$ & $<0.001$ \\
\hline $\mathrm{BMI}, \mathrm{kg} / \mathrm{m}^{2 \ddagger}$ & $24.90 \pm 3.05$ & $25.33 \pm 3.11$ & $23.77 \pm 2.57$ & $<0.001$ \\
\hline $\mathrm{SBP}, \mathrm{mm} / \mathrm{Hg}^{\ddagger}$ & $127.71 \pm 18.13$ & $128.68 \pm 18.22$ & $125.65 \pm 17.66$ & 0.025 \\
\hline $\mathrm{DBP}, \mathrm{mm} / \mathrm{Hg}^{\ddagger}$ & $77.10 \pm 10.52$ & $77.94 \pm 10.69$ & $75.34 \pm 9.99$ & 0.001 \\
\hline Alb, $g / L^{\S}$ & $45.60(42.13-47.90)$ & $45.60(42.3-48.0)$ & $45.50(41.7-47.4)$ & 0.211 \\
\hline Fasting glucose, $\mathrm{mmol} / \mathrm{L}^{\S}$ & $4.75(4.39-5.27)$ & $4.80(4.41-5.35)$ & $4.65(4.21-5.10)$ & $<0.001$ \\
\hline Insulin, $\mu \mathrm{U} / \mathrm{mL}^{\S}$ & $9.74(6.91-13.75)$ & $10.39(7.52-14.66)$ & $7.90(5.64-10.34)$ & $<0.001$ \\
\hline LDL-C, $\mathrm{mmol} / \mathrm{L}^{\ddagger}$ & $3.64 \pm 0.91$ & $3.66 \pm 0.91$ & $3.59 \pm 0.90$ & 0.301 \\
\hline $\mathrm{UA}, \mu \mathrm{mol} / \mathrm{L}^{\S}$ & 343.18 (296.37-401.18) & $350.36(301.00-409.71)$ & $325.26(288.48-373.52)$ & $<0.001$ \\
\hline$A L T, U / L^{\S}$ & $16.00(12.00-22.00)$ & $17.00(13.00-23.00)$ & $14.00(12.00-19.00)$ & $<0.001$ \\
\hline AST, $U / L^{\S}$ & $18.00(16.00-22.00)$ & $18.00(16.00-22.00)$ & $18.00(15.00-21.00)$ & 0.486 \\
\hline Physical activities, MET/day ${ }^{\S}$ & $23.21(19.85-27.66)$ & $23.26(19.97-27.63)$ & $22.75(19.75-27.90)$ & 0.886 \\
\hline Current smoking, ${ }^{\dagger} n(\%)$ & $75(8.3)$ & $51(7.7)$ & $24(9.7)$ & 0.330 \\
\hline Current drinking, ${ }^{\dagger} n(\%)$ & $73(8.0)$ & $57(8.6)$ & $16(6.5)$ & 0.290 \\
\hline Hypertension, ${ }^{+} n(\%)$ & $299(32.9)$ & $230(34.8)$ & $69(27.9)$ & 0.142 \\
\hline Diabetes, ${ }^{\dagger} n(\%)$ & $70(7.7)$ & $54(8.2)$ & $16(6.5)$ & 0.566 \\
\hline Dyslipidemia, ${ }^{\dagger} n(\%)$ & $398(43.8)$ & $310(46.9)$ & $88(35.6)$ & 0.001 \\
\hline \multicolumn{5}{|c|}{ Use of antihypertensive agents, ${ }^{+} n / N(\%)$} \\
\hline Always & 229/299 (76.6) & $173 / 230(75.2)$ & $56 / 69(81.2)$ & \\
\hline Sometimes & 0 & 0 & 0 & \\
\hline Seldom & $21 / 70(30.0)$ & $15 / 53(28.3)$ & $6 / 17(35.3)$ & \\
\hline \multicolumn{5}{|c|}{ Use of lipid-lowering agents, ${ }^{\dagger} n / N(\%)$} \\
\hline Always & $91 / 398(22.9)$ & $67 / 310(21.6)$ & $24 / 88(27.3)$ & \\
\hline Usually & $7 / 398(1.8)$ & $7 / 310(2.3)$ & 0 & \\
\hline Often & $13 / 398(3.3)$ & $11 / 310(3.5)$ & $2 / 88(2.3)$ & 0.393 \\
\hline Sometimes & $22 / 398(5.5)$ & $19 / 310(6.1)$ & $3 / 88(3.4)$ & \\
\hline Seldom & $265 / 398(66.6)$ & $206 / 310(66.5)$ & $59 / 88(67)$ & \\
\hline
\end{tabular}

BMI, body mass index; WHR, waist-to-hip ratio; SBP, systolic blood pressure; DBP, diastolic blood pressure; Alb, albumin; HOMA-IR, homeostasis model assessment of insulin resistance; TC, total cholesterol; TG, triglyceride; HDL-C, high-density lipoprotein cholesterol; LDL-C, low-density lipoprotein cholesterol; $\mathrm{UA}$, uric acid; ALT, alanine aminotransferase; AST, aspartate aminotransferase; MET, metabolic equivalent. ${ }^{\dagger}$ Categorical variables are shown as numbers and percentages and compared using the $x^{2}$ test. ${ }^{\ddagger}$ Normally distributed data were expressed as means and standard deviations and compared using Student's $t$ tests. ${ }^{5}$ Non-normally distributed data were expressed as the median and 25 th-75th interquartile range and compared using the Kruskal-Wallis test.

Inverse Association of Serum Adipsin with the Remission of NAFLD
Ann Nutr Metab 2022;78:21-32

DOI: $10.1159 / 000520368$ 


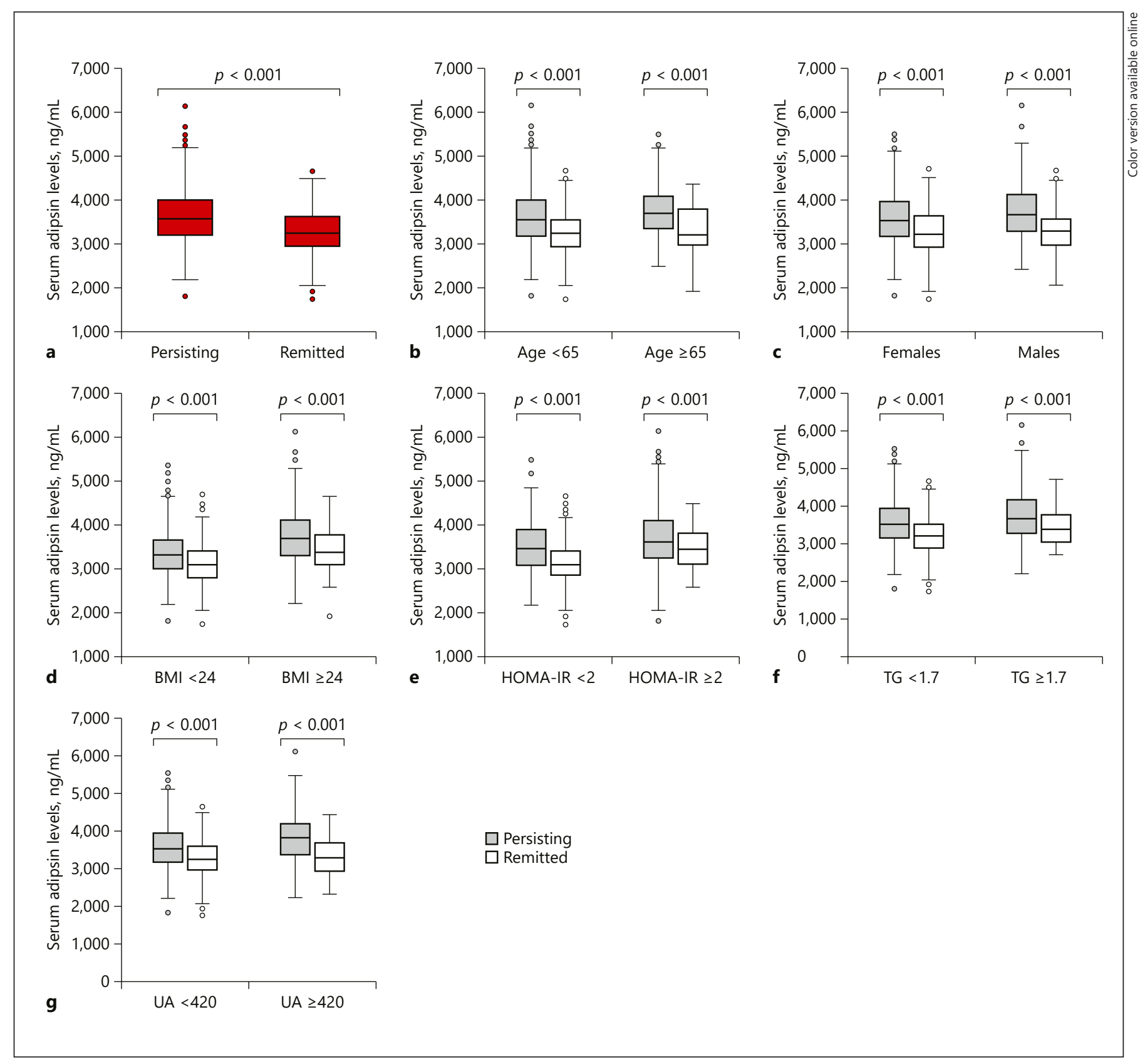

Fig. 2. Serum adipsin concentrations $(\mathrm{ng} / \mathrm{mL})$ in study participants. a Serum adipsin levels $(\mathrm{ng} / \mathrm{mL})$ in participants with persisting NAFLD and NAFLD remission. b Serum adipsin levels (ng/ $\mathrm{mL}$ ) according to age (age $<65$ and age $\geq 65$ ). c Serum adipsin levels (ng/mL) according to sex. $\mathbf{d}$ Serum adipsin levels $(\mathrm{ng} / \mathrm{mL})$ according to BMI levels (BMI $<24 \mathrm{~kg} / \mathrm{m}^{2}$ and $\mathrm{BMI} \geq 24 \mathrm{~kg} / \mathrm{m}^{2}$ ). e Serum adipsin levels (ng/mL) according to HOMA-IR (HOMA-IR $<2$ and HOMA-IR $\geq 2$ ). f Serum adipsin levels (ng/mL) according to TGs (TG $<1.7 \mathrm{mmol} / \mathrm{L}$ and $\mathrm{TG} \geq 1.7 \mathrm{mmol} / \mathrm{L}$ ). g Serum adipsin levels ( $\mathrm{ng} / \mathrm{mL}$ ) according to UA (UA $<420 \mu \mathrm{mol} / \mathrm{L}$ and UA $\geq 420$ $\mu \mathrm{mol} / \mathrm{L})$. 'Student's $t$ tests were used. ${ }^{\ddagger}$ The box plots display the median values and 25th and 75th percentiles; the whiskers represent 25 th percentiles $-1.5 \times$ interquartile range and 75 th percentiles $+1.5 \times$ interquartile range. NAFLD, nonalcoholic fatty-liver disease; BMI, body mass index; HOMA-IR, homeostasis model assessment of insulin resistance; TG, triglyceride; UA, uric acid. 
Table 2. Multivariable-adjusted ORs and $95 \% \mathrm{Cl}$ for the remission of NAFLD according to the quartiles of serum adipsin levels ${ }^{\dagger}$

\begin{tabular}{|c|c|c|c|c|c|}
\hline & \multicolumn{4}{|c|}{ Quartiles of serum adipsin levels, $\mathrm{ng} / \mathrm{mL}$} & \multirow{2}{*}{$\begin{array}{l}p \text { for } \\
\text { trend }\end{array}$} \\
\hline & Q1 & Q2 & Q3 & Q4 & \\
\hline Total $(n=908)$ & $\leq 3,115.71$ & $3,115.72-3,476.33$ & $3,476.34-3,920.18$ & $\geq 3,920.19$ & \\
\hline \multirow{2}{*}{$\begin{array}{l}\text { Model } 1^{\ddagger} \\
p \text { values }\end{array}$} & 1 & $0.57(0.39-0.84)$ & $0.27(0.18-0.42)$ & $0.17(0.11-0.27)$ & $<0.001$ \\
\hline & & 0.004 & $<0.001$ & $<0.001$ & \\
\hline \multirow{2}{*}{$\begin{array}{l}\text { Model } 2^{\S} \\
p \text { values }\end{array}$} & 1 & $0.66(0.44-0.98)$ & $0.35(0.23-0.55)$ & $0.23(0.14-0.38)$ & $<0.001$ \\
\hline & & 0.037 & $<0.001$ & $<0.001$ & \\
\hline Model $3^{\natural}$ & 1 & $0.69(0.45-1.04)$ & $0.40(0.25-0.65)$ & $0.27(0.16-0.46)$ & $<0.001$ \\
\hline$p$ values & & 0.075 & $<0.001$ & $<0.001$ & \\
\hline Female $(n=622)$ & $\leq 3,090.87$ & $3,090.88-3,442.50$ & $3,442.51-3,865.89$ & $\geq 3,865.90$ & \\
\hline \multirow{2}{*}{ Model $1^{\ddagger}$} & 1 & $0.57(0.36-0.89)$ & $0.30(0.18-0.49)$ & $0.19(0.11-0.34)$ & $<0.001$ \\
\hline & & 0.014 & $<0.001$ & $<0.001$ & \\
\hline \multirow[t]{2}{*}{ Model $2^{\S}$} & 1 & $0.63(0.39-1.01)$ & $0.39(0.23-0.66)$ & $0.27(0.15-0.51)$ & $<0.001$ \\
\hline & & 0.054 & $<0.001$ & $<0.001$ & \\
\hline \multirow[t]{2}{*}{ Model 3" } & 1 & $0.66(0.41-1.1)$ & $0.45(0.26-0.80)$ & $0.30(0.16-0.59)$ & 0.002 \\
\hline & & 0.104 & 0.006 & $<0.001$ & \\
\hline \multirow{3}{*}{$\begin{array}{l}\text { Male }(n=286) \\
\quad \text { Model } 1^{\ddagger}\end{array}$} & $\leq 3,183.11$ & $3,183.11-3,538.32$ & $3,538.33-4,010.86$ & $\geq 4,010.87$ & \\
\hline & 1 & $0.58(0.28-1.17)$ & $0.23(0.10-0.51)$ & $0.13(0.06-0.31)$ & $<0.001$ \\
\hline & & 0.13 & $<0.001$ & $<0.001$ & \\
\hline \multirow[t]{2}{*}{ Model $2^{\S}$} & 1 & $0.81(0.37-1.77)$ & $0.30(0.12-0.71)$ & $0.17(0.07-0.44)$ & $<0.001$ \\
\hline & & 0.596 & 0.007 & $<0.001$ & \\
\hline \multirow[t]{2}{*}{ Model 3 } & 1 & $0.93(0.40-2.15)$ & $0.34(0.13-0.88)$ & $0.23(0.09-0.62)$ & 0.006 \\
\hline & & 0.861 & 0.026 & 0.004 & \\
\hline \multirow{3}{*}{$\begin{array}{l}\mathrm{BMI}<24 \mathrm{~kg} / \mathrm{m}^{2}(n=360) \\
\quad \text { Model } 1^{\ddagger}\end{array}$} & $\leq 2,945.43$ & $2,945.44-3,252.90$ & $3,252.91-3,604.77$ & $\geq 3,604.78$ & \\
\hline & 1 & $0.57(0.34-0.96)$ & $0.35(0.18-0.67)$ & $0.27(0.13-0.56)$ & $<0.001$ \\
\hline & & 0.034 & 0.002 & $<0.001$ & \\
\hline \multirow[t]{2}{*}{ Model $2^{\S}$} & 1 & $0.55(0.32-0.95)$ & $0.30(0.15-0.60)$ & $0.28(0.13-0.59)$ & $<0.001$ \\
\hline & & 0.032 & 0.001 & 0.001 & \\
\hline \multirow[t]{2}{*}{ Model 3ๆ } & 1 & $0.63(0.36-1.13)$ & $0.44(0.21-0.93)$ & $0.31(0.13-0.72)$ & 0.024 \\
\hline & & 0.122 & 0.032 & 0.006 & \\
\hline \multirow{3}{*}{$\begin{array}{l}\mathrm{BMI} \geq 24 \mathrm{~kg} / \mathrm{m}^{2}(n=548) \\
\quad \text { Model } 1^{\ddagger}\end{array}$} & $\leq 3,273.63$ & $3,273.64-3,630.70$ & $3,630.71-4,045.73$ & $\geq 4,045.74$ & \\
\hline & 1 & $0.68(0.38-1.24)$ & $0.32(0.17-0.59)$ & $0.19(0.10-0.36)$ & $<0.001$ \\
\hline & & 0.21 & $<0.001$ & $<0.001$ & \\
\hline \multirow[t]{2}{*}{ Model $2^{\S}$} & 1 & $0.69(0.38-1.30)$ & $0.32(0.17-0.60)$ & $0.19(0.10-0.38)$ & $<0.001$ \\
\hline & & 0.23 & $<0.001$ & $<0.001$ & \\
\hline \multirow[t]{2}{*}{ Model 3ๆ } & 1 & $0.59(0.30-1.15)$ & $0.30(0.15-0.59)$ & $0.21(0.10-0.44)$ & $<0.001$ \\
\hline & & 0.119 & 0.001 & $<0.001$ & \\
\hline
\end{tabular}

NAFLD, nonalcoholic fatty-liver disease; $\mathrm{BMI}$, body mass index; $\mathrm{Q}$, quartile; $\mathrm{OR}$, odds ratio; $\mathrm{Cl}$, confidence interval; Alb, albumin; HOMA-IR, homeostasis model assessment of insulin resistance; TG, triglyceride; HDL-C, high-density lipoprotein cholesterol; LDL-C, low-density lipoprotein cholesterol; UA, uric acid; ALT, alanine aminotransferase; AST, aspartate aminotransferase; MET, metabolic equivalent. ${ }^{\dagger}$ Logistic regression analysis was used. ${ }^{\ddagger}$ Model 1 : adjusted for age and sex. Adjusted for age only when stratified the population by sex. ${ }^{\S}$ Model 2: adjusted for variables in model 1 plus BMI, physical activities (MET), current smoking and drinking, hypertension, and diabetes. Adjusted for variables in model 1 plus physical activities (MET), current smoking and drinking, hypertension, and diabetes when stratified the population by BMI. " Model 3: adjusted for variables in model 2 plus HOMA-IR, Alb, TG, LDL-C/HDL-C ratio, UA, and AST/ALT ratio.

mentary Table 1 (for all online suppl. material, see www. karger.com/doi/10.1159/000520368) shows that the higher the quartile grade at baseline, the fewer participants remitted to normal. Remission rates reduced from
$41.3 \%$ to $29.6 \%, 17.4 \%$, and $11.7 \%$ in participants from quartile 1 (Q1) to quartile 4 (Q4) of adipsin levels, respectively ( $p$ trend $<0.001$ ). In addition, participants in the highest quartile had higher age, BMI, WHR, systolic 
Table 3. Multiple stepwise logistic regression analyses of factors predicting remission NAFLD during the follow-up ${ }^{\dagger}$

\begin{tabular}{lllllr}
\hline Variables & $\begin{array}{l}\text { Partial regression } \\
\text { coefficient }\end{array}$ & $\begin{array}{l}\text { Standard } \\
\text { error }\end{array}$ & Wald $x^{2}$ & OR $(95 \% \mathrm{Cl})$ & $p$ value \\
\hline Adipsin & - & - & 29.08 & - & $<0.001$ \\
Q1 & - & - & - & - & - \\
Q2 & -0.42 & 0.21 & 4.15 & $0.66(0.44-0.98)$ & 0.042 \\
Q3 & -0.91 & 0.23 & 15.54 & $0.40(0.26-0.63)$ & $<0.001$ \\
Q4 & -1.26 & 0.26 & 23.80 & $0.28(0.17-0.47)$ & $<0.001$ \\
BMI, kg/m ${ }^{2}$ & -0.12 & 0.03 & 12.78 & $0.89(0.84-0.95)$ & $<0.001$ \\
TGs, $\mathrm{mmol} / \mathrm{L}$ & -0.55 & 0.11 & 23.99 & $0.58(0.46-0.72)$ & $<0.001$ \\
AST/ALT ratio & 0.57 & 0.20 & 8.41 & $1.77(1.20-2.60)$ & 0.004 \\
Constant & 2.58 & 0.87 & 8.78 & 13.23 & 0.003 \\
\hline
\end{tabular}

Variables in original model were baseline age, sex, BMI, physical activity (METs/day), HOMA-IR, fasting glucose, AST/ALT ratio, TC, LDL-C/HDL-C ratio, Trunk fat percentage (\%), UA, Alb, and adipsin levels in quartiles. OR, odds ratio; $\mathrm{Cl}$, confidence interval; $\mathrm{Q}$, quartile; BMI, body mass index; Alb, albumin; HOMA-IR, homeostasis model assessment of insulin resistance; TC, total cholesterol; HDL-C, high-density lipoprotein cholesterol; LDL-C, lowdensity lipoprotein cholesterol; UA, uric acid; ALT, alanine aminotransferase; AST, aspartate aminotransferase; MET, metabolic equivalent. blood pressure, diastolic blood pressure, fasting glucose, TGs, insulin, HOMA-IR, UA, ALT, trunk fat percentage, hypertension, and diabetes prevalence and lower levels of Alb and HDL-C than those in the lowest quartile (all $p<$ $0.05)$.

\section{Serum Adipsin Level Is Positively Correlated with \\ $B M I$, Insulin, and HOMA-IR}

The correlation between baseline serum adipsin levels and clinical parameters was analyzed. Correlation coefficients are shown in online supplementary Table 2. Significant positive correlations were observed between adipsin levels and BMI $(r: 0.38, p<0.001)$, insulin $(r: 0.30$, $p<0.001)$, and HOMA-IR $(r: 0.31, p<0.0001)$. After adjusting for age and sex, positive correlations of serum adipsin levels with BMI, insulin, and HOMA-IR were still observed (all $p<0.001$ ).

\section{Prospective Analyses of the Association between}

Baseline Serum Adipsin and Remission of NAFLD at the Follow-Up

Baseline serum adipsin levels were inversely associated with NAFLD remission in participants diagnosed with NAFLD at baseline (all $p<0.05$ for trend). Table 2 shows the results of logistic regression analysis. The odds ratio (OR) for the remission of NAFLD was 0.17 for participants in the highest quartile compared with those in the lowest quartile (95\% CI: $0.11-0.27, p$ for trend $<0.001)$ in model 1 . Higher serum adipsin levels were associated with an increased risk of persisting NAFLD in model 2
(OR: $0.23,95 \%$ CI: $0.14-0.38, p$ for trend $<0.001$ ) and model 3 (OR: 0.27, 95\% CI: 0.16-0.46, $p$ for trend $<0.001$ ). Analyses showed similar results in subgroups. Serum adipsin levels were also inversely associated with the remission of NAFLD in the quartiles defined sex-specific and BMI-specific.

\section{Serum Adipsin Level Independently Predicts the \\ Remission of NAFLD}

We investigated the prediction of NAFLD remission with the observed parameters using a multiple stepwise logistic regression model (Table 3 ). According to the ORs, 6 variables were significantly and independently related to the remission of NAFLD, including baseline adipsin quartile $2(\mathrm{Q} 2)$, adipsin quartile $3(\mathrm{Q} 3)$, adipsin Q4, BMI, TGs, and AST/ALT ratio (all $p<0.05$ ). Adipsin Q2, adipsin Q3, adipsin Q4, BMI, and TGs were inversely correlated, while the ratio was positively correlated with NAFLD remission. Based on the above results, we established and named the model for predicting NAFLD remission the Adipsin Model for NAFLD Remission, which is calculated as follows: $\mathrm{e}^{\mathrm{M}} /\left(1+\mathrm{e}^{\mathrm{M}}\right), M=2.58-0.42$ $\times$ adipsin Q2 (vs. Q1) - $0.91 \times$ adipsin Q3 (vs. Q1) - 1.26 $\times$ adipsin Q4 (vs. Q1) $-0.12 \times \mathrm{BMI}\left(\mathrm{kg} / \mathrm{m}^{2}\right)-0.55 \times \mathrm{TGs}$ $(\mathrm{mmol} / \mathrm{L})+0.57 \times$ AST/ALT ratio.

\section{Evaluation of Serum Adipsin as a Predictor for NAFLD Remission}

The receiver operating characteristic curves shown in Figure 3 represent the prediction accuracy of serum
28

Ann Nutr Metab 2022;78:21-32 DOI: $10.1159 / 000520368$
Gu/Luo/Chen/Qiu/Zhou/Wang/Qian/ Liu/Xie/Xu/Ling/Chen/Yang 
Table 4. Sensitivity, specificity, positive likelihood ratio, and negative likelihood ratio for NAFLD remission prediction with serum adipsin and Adipsin Model for NAFLD Remission ${ }^{\dagger}$

\begin{tabular}{|c|c|c|c|c|c|c|}
\hline Predictor & $\begin{array}{l}\text { Cutoff values } \\
\text { of the SHBG mode }\end{array}$ & $\begin{array}{l}\text { Sensitivity, } \\
\%\end{array}$ & $\begin{array}{l}\text { Specificity, } \\
\%\end{array}$ & $\begin{array}{l}\text { Positive } \\
\text { likelihood ratio }\end{array}$ & $\begin{array}{l}\text { Negative } \\
\text { likelihood ratio }\end{array}$ & $p$ value \\
\hline \multicolumn{7}{|l|}{ Serum adipsin } \\
\hline 16th percentile & & 0.883 & 0.300 & 0.618 & 0.035 & \multirow{3}{*}{$<0.001$} \\
\hline 26th percentile & & 0.709 & 0.578 & 0.299 & 0.168 & \\
\hline 39th percentile & & 0.413 & 0.811 & 0.078 & 0.476 & \\
\hline \multicolumn{7}{|c|}{ Adipsin Model for NAFLD Remission } \\
\hline 15 th & & 0.931 & 0.384 & 0.358 & 0.042 & \multirow{8}{*}{$<0.001$} \\
\hline 25th & & 0.749 & 0.638 & 0.478 & 0.091 & \\
\hline 35 th & & 0.575 & 0.782 & 0.450 & 0.093 & \\
\hline 45th & & 0.332 & 0.890 & 0.295 & 0.074 & \\
\hline 55 th & & 0.154 & 0.949 & 0.146 & 0.044 & \\
\hline 65 th & & 0.061 & 0.988 & 0.060 & 0.011 & \\
\hline 75th & & 0.004 & 0.998 & 0.004 & 0.002 & \\
\hline 86th & & 0.000 & 0.998 & 0.000 & 0.002 & \\
\hline
\end{tabular}

NAFLD, nonalcoholic fatty-liver disease; ROC, receiver operating characteristic. ${ }^{\dagger} \mathrm{ROC}$ curve analyses were used.

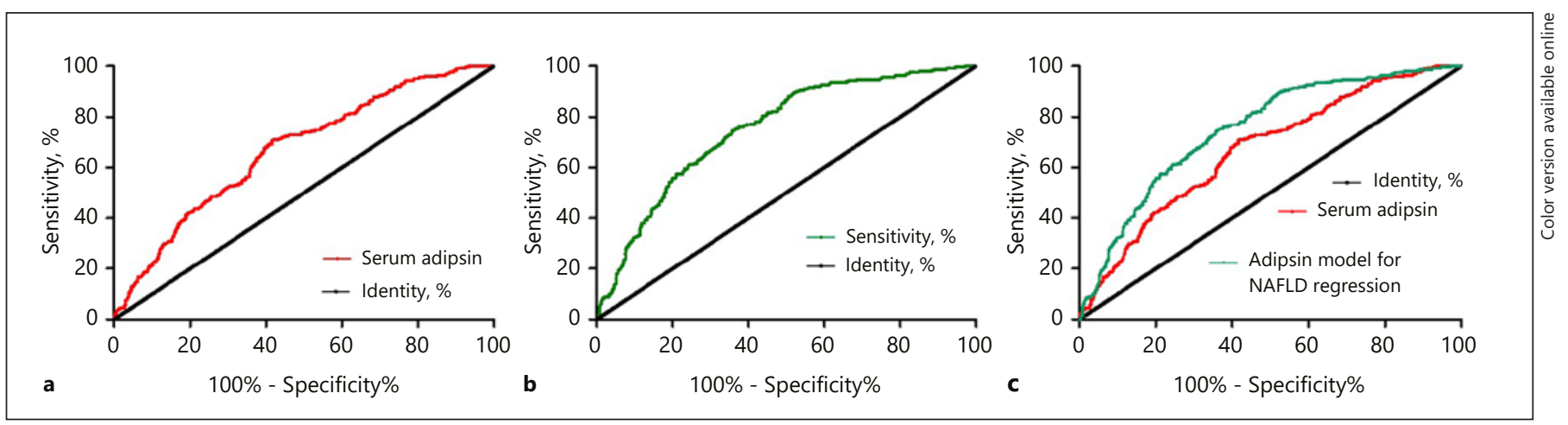

Fig. 3. The ROC curves of serum adipsin and the Adipsin Model for NAFLD Remission developed by ROC curve analyses. Prediction of NAFLD remission by serum adipsin (a) and the Adipsin Model for NAFLD Remission (b). c AUROC for the model was larger than that for serum adipsin $(p<0.001) . p<0.001$ for all ROC curves compared with the 0.5 curve. NAFLD, nonalcoholic fatty-liver disease; AUROC, receiver operating characteristic curve; ROC, receiver operating characteristic. adipsin and the Adipsin Model for NAFLD Remission. The AUROC for the model was larger (AUROC: 0.751, 95\% CI: $0.717-0.785)(p<0.001)$ than that for serum adipsin alone (AUROC: 0.673, 95\% CI: 0.634-0.712) ( $p$ $<0.001)$. The optimal cutoff values of serum adipsin level and the model were located at 25.6th percentile and 25.1 th percentile, which corresponded to $70.9 \%$ sensitivity and $57.8 \%$ specificity for adipsin and $74.9 \%$ sensitivity and $64.0 \%$ specificity for the model, respectively (Table 4).

Inverse Association of Serum Adipsin with the Remission of NAFLD

\section{Discussion}

In this study, using a community-based population with a 3-year prospective follow-up, we showed for the first time the association between serum adipsin levels and the remission of NAFLD in middle-aged and elderly Chinese adults. Our results indicate that adipsin levels are inversely correlated with the remission of NAFLD and support the hypothesis that serum adipsin is an independent predictor for the remission of NAFLD. 
Growing evidence suggests a link between metabolic diseases and adipsin. Several human studies have demonstrated that the levels of adipsin are higher in metabolic diseases associated with obesity [18, 20,27-29]. In a study by Calan et al. [18], circulating adipsin levels were elevated in overweight/obese women compared with lean women, with a positive correlation between adipsin and BMI, HOMA-IR, and TGs in polycystic ovary syndrome women and their controls. It has also been reported that adipsin increases TG synthesis [30,31]. Our recent study showed a positive association of circulating adipsin levels with the presence of NAFLD in Chinese adults [23]. These research results are coherent with our current results that circulating adipsin levels were positively correlated with BMI and TGs. However, a case-control study conducted by Yilmaz et al. [21] found that serum adipsin levels did not differ between participants with NAFLD and the control group. Moreover, no significant difference in adipsin levels was observed among children with different severities of NAFLD [22]. One study reported that adipsin mRNA expression and circulating adipsin levels significantly decreased in obese mice caused by genetic or metabolic defects [32]. These discrepancies may be due to the different age-groups, relatively small sample sizes, and the limitations of cross-sectional studies in human studies or species differences between humans and mice.

Adipokine alterations, which occur during the expansion of adipose tissue, are involved in the pathogenesis of NAFLD and can promote the development of SS-, NASH, and NASH-related cirrhosis $[33,34]$. NAFLD is the hepatic manifestation of obesity and metabolic syndrome, as well as a dynamic condition in which severe NAFLD can remit to simple steatosis or normal liver. Thus, identifying noninvasive biomarkers to predict the remission of NAFLD to decrease liver disease-related mortality is urgent. In this study, we found that participants with subsequent NAFLD remission had lower serum adipsin levels than those who had persisting NAFLD. Logistic regression analysis revealed that decreased circulating adipsin levels were significantly associated with high odds of NAFLD remission, and the association remained the same in subgroups. Multiple stepwise logistic regression analysis proved that adipsin independently predicted the remission of NAFLD. Our findings highlight that adipsin may act as a detrimental factor during the remission of NAFLD, which could potentially be an indicator of the remission of NAFLD. To the best of our knowledge, our study provided the first clinical evidence on the association between adipsin and NAFLD in a prospective cohort study.
Adipsin is also known as complement factor D, which is a key component of the alternative complement pathway [12]. The immune system plays a significant role in the pathogenesis of NAFLD. Rensen et al. [16] found that most NAFLD participants presented hepatic activated C3 deposition, and participants showing activated C3 deposition displayed increased numbers of apoptotic cells and hepatic inflammatory infiltration, and they were more likely to develop NASH. In the study of Segers et al. [17], alternative pathway activation played an important role in driving hepatic inflammation in NASH. Likewise, Jia et al. [35] reported that increased serum C3 levels were significantly associated with a high risk of NAFLD. Upregulation of adipsin is related to the progression of steatosis to spontaneous IR, NAFLD, and HCC in mice [36]. In addition to adipocytes, HepG2 cells can also synthesize complement protein $\mathrm{D}$ and secrete high levels of factor $\mathrm{D}$ into culture medium $[37,38]$, but this biological effect is not clear.

Our study has limitations that should be taken into consideration. First, up to July 2016, more than onethird of the participants with NAFLD from the baseline survey had not completed the follow-up survey. However, most of the demographic, anthropometric, and biochemical parameters of those participants did not differ from those of the participants who completed the follow-up survey. Therefore, the risk for selection bias may be excluded. In addition, the diagnosis of NAFLD was determined noninvasively, therefore raising concerns about reduced sensitivity and specificity in NAFLD diagnosis compared to liver biopsy. Moreover, there were more than twice as many women as men in the study. To reduce the potential bias, participants were stratified by sex, and the inverse association between adipsin and the remission of NAFLD was still observed in the subgroup analysis.

\section{Conclusions}

In conclusion, baseline circulating adipsin levels were lower in NAFLD remission participants in this prospective cohort study performed in the Chinese population. Serum adipsin can be a potential biomarker for predicting NAFLD remission. Although the physiological roles of adipsin in NAFLD have not been elucidated, it is reasonable to speculate that adipsin might play a vital pathophysiological role in NAFLD based on the results of the 3-year community-based cohort study. In the future, prospective studies involving larger sample sizes of multieth-
Ann Nutr Metab 2022;78:21-32 DOI: $10.1159 / 000520368$
Gu/Luo/Chen/Qiu/Zhou/Wang/Qian/ Liu/Xie/Xu/Ling/Chen/Yang 
nic populations of all ages with NAFLD diagnosed and assessed by transient elastography, magnetic resonance spectroscopy, or biopsy are needed.

\section{Acknowledgments}

The authors thank all participants who participated in this study.

\section{Statement of Ethics}

The GNHS was registered at ClinicalTrials.gov (Registration Number NCT03179657) and approved by the Ethics Committee of the School of Public Health at Sun Yat-sen University (approval number ZDGWYL2009-3), which meets the guidelines laid down in the Declaration of Helsinki. Written informed consent was obtained from all participants.

\section{Conflict of Interest Statement}

The authors declare that there are no conflicts of interest.

\section{Funding Sources}

This work was supported by the State Key Program of National Natural Science Foundation of China (Grant No. 81730090) and National Natural Science Foundation of China (81872613).

\section{Author Contributions}

Wenhua Ling, Yuming Chen, and Lili Yang contributed to study concept and design. Xiaoyun Qian, Yao Liu, Jiewen Xie, Zhongliang Xu, Qian Chen, Yun Qiu, Yujia Zhou, Xu Wang, Yingying $\mathrm{Gu}$, and Jing Luo conducted the study. Yingying $\mathrm{Gu}$ and Jing Luo contributed to data collection and analysis. All the authors contributed to critical review and revision of the manuscript for important intellectual content.

\section{Data Availability Statement}

Data are available from the corresponding author upon reasonable request.

\section{References}

1 Younossi Z, Henry L. Contribution of alcoholic and nonalcoholic fatty liver disease to the burden of liver-related morbidity and mortality. Gastroenterology. 2016;150(8): 1778-85.

2 Chalasani N, Younossi Z, Lavine JE, Charlton $\mathrm{M}$, Cusi K, Rinella M, et al. The diagnosis and management of nonalcoholic fatty liver disease: practice guidance from the American Association for the study of liver diseases. Hepatology. 2018;67(1):328-57.

3 Satapathy SK, Sanyal AJ. Epidemiology and natural history of nonalcoholic fatty liver disease. Semin Liver Dis. 2015;35(3):221-35.

4 Younossi Z, Henry L. Contribution of alcoholic and nonalcoholic fatty liver disease to the burden of liver-related morbidity and mortality. Gastroenterology. 2016;150(8): 1778.

5 Hausman DB, DiGirolamo M, Bartness TJ, Hausman GJ, Martin RJ. The biology of white adipocyte proliferation. Obes Rev. 2001;2: 239-54.

6 Kershaw EE, Flier JS. Adipose tissue as an endocrine organ. J Clin Endocrinol Metab. 2004;89(6):2548-56.

7 Jung UJ, Choi MS. Obesity and its metabolic complications: the role of adipokines and the relationship between obesity, inflammation, insulin resistance, dyslipidemia and nonalcoholic fatty liver disease. Int J Mol Sci. 2014; 15(4):6184-223.
8 Cook KS, Min HY, Johnson D, Chaplinsky RJ, Flier JS, Hunt CR, et al. Adipsin: a circulating serine protease homolog secreted by adipose tissue and sciatic nerve. Science. 1987;237: 402-5.

9 Baldo A, Sniderman AD, St-Luce S, Avramoglu RK, Maslowska M, Hoang B, et al. The adipsin-acylation stimulating protein system and regulation of intracellular triglyceride synthesis. J Clin Invest. 1993;92:1543-7.

10 Cianflone K, Roncari DA, Maslowska M, Baldo A, Forden J, Snidermant AD. Adipsin/acylation stimulating protein system in human adipocytes: regulation of triacylglycerol synthesis? Biochemistry. 1994;33:9489-95.

11 Lisa N, Choy BR, Spiegelman BM. Adipsin and an endogenous pathway of complement from adipose cells. J Biol Chem. 1992;297: 12736-41.

12 White RT, Damm D, Hancock N, Rosen BS, Lowell BB, Usher PJ, et al. Human adipsin is identical to complement factor D and is expressed at high levels in adipose tissue. J Biol Chem. 1992;267:9210-3.

$13 \mathrm{Xu}$ Y, Ma M, Ippolito GC, Schroeder HW Jr, Carroll MC, Volanakis JE. Complement activation in factor D-deficient mice. Proc Natl Acad Sci U S A. 2001;98(25):14577-82.

14 Shahini N, Michelsen AE, Nilsson PH, Ekholt $\mathrm{K}$, Gullestad L, Broch K, et al. The alternative complement pathway is dysregulated in patients with chronic heart failure. Sci Rep. 2017;7:42532.
15 Moreno-Navarrete JM, Fernández-Real JM. The complement system is dysfunctional in metabolic disease: evidences in plasma and adipose tissue from obese and insulin resistant subjects. Semin Cell Dev Biol. 2019;85: $164-72$.

16 Rensen SS, Slaats Y, Driessen A, Peutz-Kootstra CJ, Nijhuis J, Steffensen R, et al. Activation of the complement system in human nonalcoholic fatty liver disease. Hepatology. 2009;50(6):1809-17.

17 Segers FM, Verdam FJ, de Jonge C, Boonen B, Driessen A, Shiri-Sverdlov R, et al. Complement alternative pathway activation in human nonalcoholic steatohepatitis. PLoS One. 2014;9(10):e110053.

18 Gursoy Calan O, Calan M, Yesil Senses P, Unal Kocabas G, Ozden E, Sari KR, et al. Increased adipsin is associated with carotid intima media thickness and metabolic disturbances in polycystic ovary syndrome. Clin Endocrinol. 2016;85(6):910-7.

19 Napolitano A, Lowell BB, Damm D, Leibel RL, Ravussin E, Jimerson DC, et al. Concentrations of adipsin in blood and rates of adipsin secretion by adipose tissue in humans with normal, elevated and diminished adipose tissue mass. Int J Obes Relat Metab Disord. 1994;18:213-8
Inverse Association of Serum Adipsin with the Remission of NAFLD
Ann Nutr Metab 2022;78:21-32

DOI: $10.1159 / 000520368$ 
20 Pomeroy C, Mitchell J, Eckert E, Raymond N, Crosby R, Dalmasso AP. Effect of body weight and caloric restriction on serum complement proteins, includilng factor $\mathrm{D}$ /adipsin: studies in anorexia nervosa and obesity. Clin Exp Immunol. 1997;108:507-15.

21 Yilmaz Y, Yonal O, Kurt R, Alahdab YO, Eren F, Ozdogan O, et al. Serum levels of omentin, chemerin and adipsin in patients with biopsyproven nonalcoholic fatty liver disease. Scand J Gastroenterol. 2011;46(1):91-7.

22 Fitzpatrick E, Dew TK, Quaglia A, Sherwood RA, Mitry RR, Dhawan A. Analysis of adipokine concentrations in paediatric non-alcoholic fatty liver disease. Pediatr Obes. 2012; 7(6):471-9.

23 Qiu Y, Wang SF, Yu C, Chen Q, Jiang R, Pei $\mathrm{L}$, et al. Association of circulating adipsin, visfatin, and adiponectin with nonalcoholic fatty liver disease in adults: a case-control study. Ann Nutr Metab. 2019;74(1):44-52.

24 Chen ZY, Liu M, Jing LP, Xiao ML, Dong HL, Chen GD, et al. Erythrocyte membrane n-3 polyunsaturated fatty acids are inversely associated with the presence and progression of nonalcoholic fatty liver disease in Chinese adults: a prospective study. Eur J Nutr. 2020; 59(3):941-51.

25 Luo J, Chen Q, Shen T, Wang X, Fang W, Wu $\mathrm{X}$, et al. Association of sex hormone-binding globulin with nonalcoholic fatty liver disease in Chinese adults. Nutr Metab. 2018;15:79.
26 Ainsworth BE, Haskell WL, Herrmann SD, Meckes N, Bassett DR Jr, Tudor-Locke C, et al. Compendium of physical activities: a second update of codes and MET values. Med Sci Sports Exerc. 2011;43(8):1575-81.

27 Vasilenko MA, Kirienkova EV, Skuratovskaia DA, Zatolokin PA, Mironyuk NI, Litvinova LS. The role of production of adipsin and leptin in the development of insulin resistance in patients with abdominal obesity. Dokl Biochem Biophys. 2017;475(1):271-6.

28 Gomez-Banoy N, Guseh JS, Li G, Rubio-Navarro A, Chen T, Poirier B, et al. Adipsin preserves beta cells in diabetic mice and associates with protection from type 2 diabetes in humans. Nat Med. 2019;25(11):1739-47.

29 Ohtsuki T, Satoh K, Shimizu T, Ikeda S, Kikuchi N, Satoh T, et al. Identification of adipsin as a novel prognostic biomarker in patients with coronary artery disease. J Am Heart Assoc. 2019;8(23):e013716.

30 Cianflone K, Roncari DA, Maslowska M, Baldo A, Forden J, Sniderman AD. Adipsin/acylation stimulating protein system in human adipocytes: regulation of triacylglycerol synthesis. Biochemistry. 1994;33:9489-95.

31 Baldo AA, Sniderman AD, St-Luce S, Avramoglu RK, Maslowska M, Hoang B, et al. The adipsin-acylation stimulating protein system and regulation of intracellular triglyceride synthesis. J Clin Invest. 1993;92(3): $1543-7$.
32 Flier JS, Cook KS, Usher P, Spiegelman BM. Severely impaired adipsin expression in genetic and acquired obesity. Science. 1987;237: 405-8.

33 Polyzos SA, Kountouras J, Zavos C. Nonalcoholic fatty liver disease: the pathogenetic roles of insulin resistance and adipocytokines. Curr Mol Med. 2009;9:299-314

34 Boutari C, Tziomalos K, Athyros VG. The adipokines in the pathogenesis and treatment of nonalcoholic fatty liver disease. Hippokratia. 2016;20:259-63.

35 Jia Q, Li C, Xia Y, Zhang Q, Wu H, Du H, et al. Association between complement C3 and prevalence of fatty liver disease in an adult population: a cross-sectional study from the Tianjin Chronic Low-Grade Systemic Inflammation and Health (TCLSIHealth) cohort study. PLoS One. 2015;10(4):e0122026.

36 Hines IN, Hartwell HJ, Feng Y, Theve EJ, Hall GA, Hashway S, et al. Insulin resistance and metabolic hepatocarcinogenesis with parentof-origin effects in $\mathrm{A} \times \mathrm{B}$ mice. Am J Pathol. 2011;179(6):2855-65.

37 Barnum SR, Volanakis JE. Biosynthesis of complement protein D by HepG2 cells: a comparison of D produced by HepG2 cells, U937 cells and blood monocytes. Eur J Immunol. 1985;15(11):1148-51.

38 Kitano E, Kitamura H. Synthesis of factor D by normal human hepatocytes. Int Arch Allergy Immunol. 2000;122:299-302. 